COMMENTARY. This review provides a comprehensive classification of the clinical and genetic features of syndromes associated with callosal agenesis. In a clinical reference guide to neurological syndromes [1], among approximately 250 syndromes listed, 18 have an associated CC dysgenesis. Seizures, especially infantile spasms, as in Aicardi syndrome, are an established complication of ACC whereas behavioral and cognitive impairments as isolated deficits are less well documented.

ACC and autism. About one third of a group of 26 adults with ACC presented with autism whereas in childhood only 3 of the group had met criteria for a diagnosis of autism spectrum disorder [2]. Parent ratings of childhood behavior indicate that children with agenesis are less likely to be autistic but as adults ACC is a risk factor for developing autism. Both genetic and environmental factors may be involved in the cause of ACC and its autistic features.

\author{
References. \\ 1. Millichap JG. Neurological Syndromes : A Clinical Guide to Symptoms and Diagnosis. New York: \\ Springer; 2013:279. \\ 2. Paul LK, et al. Brain. 2014 Jun;137(Pt 6):1813-29.
}

\title{
NEUROPSYCHOLOGICAL AND LANGUAGE DEFICITS IN 22q11.2 DELETION SYNDROME
}

Investigators at Bambino Genu Children's Hospital, Rome, and multiple additional centers in Italy conducted a retrospective and prospective study of clinical manifestations at diagnosis and during follow-up of 228 patients with 22q11.2 deletion syndrome. Clinical diagnosis, confirmed by cytogenic or molecular analysis, was made before 2 years of age in $71 \%$ of patients. Median age at diagnosis was 4 months (range 0 to 36 years 10 months). Early diagnosis was predominantly related to heart anomalies and neonatal hypocalcemia. In patients diagnosed after 2 years of age, speech and language impairment, developmental delay and facial features were the main diagnostic elements. During follow-up, the frequency of autoimmune manifestations $(\mathrm{P}=0.015)$ and speech disorders $(\mathrm{P}=0.002)$ increased. Psychomotor and speech/language developmental delay were reported in $48 \%$ and $53 \%$ cases, respectively, leading to the diagnosis in subjects older than 2 years. Orthopedic abnormalities, mainly scoliosis and congenital clubfoot, were observed in 78 of 217 patients. (Cancrini C, Puliafito P, Digilio MC, et al. Clinical features and follow-up in patients with 22q11.2 deletion syndrome. J Pediatr 2014 Jun;164(6):1475-1480.e2).

COMMENTARY. The syndrome 22q11.2 deletion, also known as DiGeorge (DGS), velo-cardio-facial (VCFS), cono-truncal-anomaly-face (CTAF), Shprintzen, Strong, Sedlackova, Cayler cardiofacial, and congenital thymic aplasia syndromes, is of interest particularly to the cardiologist in patients presenting in early infancy. In children older than 2 years, the neurologist is consulted because of speech and language delay and psychomotor behavioral disorders. Symptoms of hypocalcemia may complicate heart anomalies in the neonatal period and behavioral disorders in older children. Patients of all ages share the characteristic dysmorphic features of hypertelorism, broad nose tip, small mouth, and ear anomalies. The main presenting features of 22q11.2 deletion syndrome vary with the patient's age [1]. The mnemonic, CATCH-22 is often applied to the 
diagnostic features: C-Cardiac abnormality (tetralogy of Fallot): A-Abnormal facies (hypertelorism);T-Thymic aplasia; C-Cleft palate; H-Hypocalcemia /hypoparathyroidism; 22-Chromosome abnormality [2].

\section{References.}

1. Digilio MC, et al. Clin Genet. 2003 Apr;63(4):308-13.

2. Millichap JG. Neurological Syndromes : A Clinical Guide to Symptoms and Diagnosis. New York: Springer; 2013:279.

\section{ENCEPHALOPATHIES}

\section{ANTECOLLIS AND PARKINSONISM IN ADULTS WITH DRAVET SYNDROME}

Investigators at Toronto Western and The Hospital for Sick Children, Toronto, Canada, prospectively studied the motor abnormalities in a consecutive sample of adults with genetically proven Dravet syndrome (DS). Of 12 patients entering the study, 11 presented with severe cognitive delay, and 1 had mild spasticity. All had flexion of the head and $8(66 \%)$ had dystonic antecollis, severity correlating with age. Mild parkinsonism in 11 patients $(91 \%)$ was characterized by global bradykinesia and asymmetric cogwheel rigidity. Severity of parkinsonism was correlated with age but not with seizure frequency or use and dose of valproate. Crouch gait (in 5/12 cases), small steps, and/or wide base gait impairment occurred in 9 patients. Two of 4 most affected patients experienced sustained improvement of gait following levodopa treatment and were no longer wheelchair bound. (Fasano A, Borlot F, Lang AE, et al. Antecollis and levodopa-responsive parkinsonism are late features of Dravet syndrome. Neurology 2014 Jun 17;82(24):2250-1).

COMMENTARY. The cause of the postural and motor signs in adults with Dravet syndrome is unclear. The Toronto authors favor the progressive involvement of the nigrostriatal dopamine system but admit the possibility of adverse effects of antiepileptic drugs [1]. Crouch gait in $50 \%$ of adults with DS and SCN1A mutations was observed only in those patients with nonsense mutations or mutations in the pore-forming region of the $\mathrm{Na} 1.1$ protein. Crouch gait, without spasticity, is identified as an element of the adult DS phenotype [2].

Investigators in Paris, France, discuss cognitive impairment in Dravet syndrome that it is not related to major epileptic factors (duration of seizures, fever-related status epilepticus) but more a consequence of genetic factors. SCN1A mutation directly contributes to cognitive impairment independent of seizure induction. Dravet syndrome should no longer be considered an epileptic encephalopathy [3].

\section{References.}

1. Fasano A, et al. Neurology. 2014 Jun 17;82(24):2250-1.

2. Rilstone JJ, et al. Epilepsia. 2012 Aug;53(8):1421-8.

3. Auvin S, et al. Editorial. Should we still consider Dravet syndrome an epileptic encephalopathy? Epilepsy Behav. 2014 May 31;36C:80-81. 